\title{
Molecular Epidemiology of Tuberculosis in a Sentinel Surveillance Population
}

\author{
Barbara A. Ellis, ${ }^{*}$ Jack T. Crawford, ${ }^{*}$ Christopher R. Braden, ${ }^{*}$ Scott J. N. McNabb, ${ }^{*}$ \\ Marisa Moore, ${ }^{*}$ Steve Kammerer, ${ }^{*}$ and the National Tuberculosis Genotyping and \\ Surveillance Network Work Group ${ }^{1}$
}

\begin{abstract}
We conducted a population-based study to assess demographic and risk-factor correlates for the most frequently occurring Mycobacterium tuberculosis genotypes from tuberculosis (TB) patients. The study included all incident, culture-positive TB patients from seven sentinel surveillance sites in the United States from 1996 to 2000. M. tuberculosis isolates were genotyped by IS6110-based restriction fragment length polymorphism and spoligotyping. Genotyping was available for $90 \%$ of 11,923 TB patients. Overall, $48 \%$ of cases had isolates that matched those from another patient, including $64 \%$ of U.S.-born and $35 \%$ of foreign-born patients. By logistic regression analysis, risk factors for clustering of genotypes were being male, U.S.-born, black, homeless, and infected with HIV; having pulmonary disease with cavitations on chest radiograph and a sputum smear with acid-fast bacilli; and excessive drug or alcohol use. Molecular characterization of TB isolates permitted risk correlates for clusters and specific genotypes to be described and provided information regarding cluster dynamics over time.
\end{abstract}

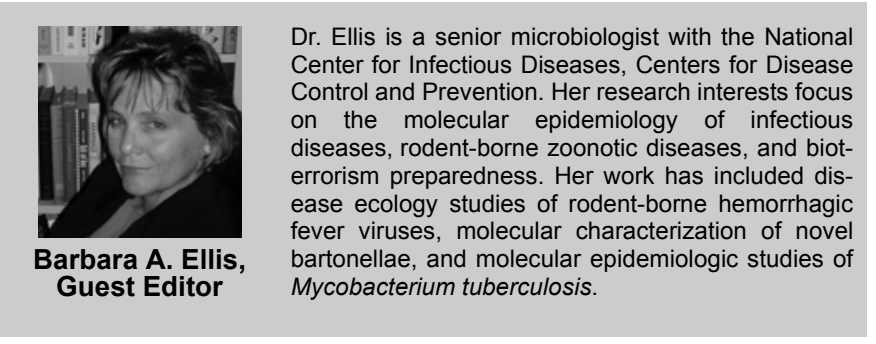

Q ince 1990, characterization of Mycobacterium tuberculosis $\checkmark$ isolates by molecular methods has been useful in confirming suspected laboratory contamination and as an adjunct to epidemiology-based contact investigation (1-3). Most studies used the restriction fragment length polymorphism (RFLP) technique, based on IS6110 and specific to the M. tuberculosis complex. This genetic element may be present in different positions on the chromosome, resulting in a unique genotype useful for characterizing the strain of $M$. tuberculosis infecting a patient. Although RFLP has disadvantages (e.g., cost, time required to culture the organism, and specialized training and laboratory equipment), IS6110-based RFLP is the established method considered most discriminatory for genetic characterization of $M$. tuberculosis strains worldwide (4).

In 1996, the Centers for Disease Control and Prevention (CDC) established seven sentinel surveillance sites in the United States (National Tuberculosis Genotyping and Surveillance Network) to assess the utility of molecular genotyping for improving tuberculosis (TB) prevention and control. The TB genotyping network used standardized protocols for molecular characterization of M. tuberculosis isolates from

${ }^{*}$ Centers for Disease Control and Prevention, Atlanta, Georgia, USA patients in all sentinel sites. The network was designed to address specific epidemiologic questions regarding the natural history, transmission, and potential applicability of molecular genotyping of $M$. tuberculosis strains to augment TB control activities (5). Two objectives were to identify and determine the prevalence of specific $M$. tuberculosis genotype clustering in populations of sentinel surveillance TB patients and to describe the demographic characteristics of these populations and the genotypic characteristics of $M$. tuberculosis strains in clustered and nonclustered TB cases. We describe demographic and risk factor correlates for the most frequently occurring M. tuberculosis genotypes in isolates collected from sentinel TB patients.

\section{Methods}

This population-based sentinel study included all incident culture-positive TB patients from sentinel sites from January 1996 to December 2000. In brief, the seven sentinel surveillance sites included the states of Arkansas, Maryland, Massachusetts, Michigan, and New Jersey; Dallas, Tarrant, Cameron, and Hidalgo Counties in Texas; and Alameda, Contra Costa, Marin, San Mateo, Santa Clara, and Solano Counties in California. A detailed description of the study's design, participants, population, and laboratory and epidemiologic methods is provided elsewhere (6).

\footnotetext{
${ }^{1}$ Members of the National Tuberculosis Genotyping and surveillance Network Work Group, in addition to the listed authors, included Joseph Bates, William Benjamin, Pablo Bifani, M. Donald Cave, Rebecca Cox, Wendy Cronin, Ed Desmond, Jeffrey Driscoll, Nancy Dunlap, Jennifer Flood, Kashef ljaz,, Michael Kucab, Barry Kreiswirth, Zary Liu, D. Mitchell Magee, Jeffrey Massey, Ann Miller, Donna Mulcahy, Robert Pratt, Teresa Quitugua, Barbara Schable, Kenneth Shilkret, Harry Taber, Jeffrey Taylor, Sharon Sharnprapai, Sumi Sun, Zhenhua Yang
} 
All patients included in the study were reported to the CDC national TB case registry on the form Report of a Verified Case of Tuberculosis, a standardized electronic form submitted for TB surveillance to CDC by all state public health reporting areas. Data reported include patient demographics, laboratory test results, drug susceptibilities, information on chest radiographs, and treatment outcomes (7).

Investigators from the sentinel surveillance sites submitted patient isolates to the corresponding regional laboratory for genotyping and conducted routine contact investigations. In addition, participants from the surveillance sites performed detailed epidemiologic investigations on groups of persons with $M$. tuberculosis isolates that had matching genetic patterns or clusters (see below). The regional genotyping laboratories conducted IS6110 RFLP on isolates from sentinel patients. Since low-copy numbers of IS6110 (i.e., six or fewer copies) reduce test specificity, spacer oligonucleotide typing (spoligotyping) was conducted on such isolates. A cluster, which was identified by analysis of the entire TB genotyping network database, was defined as two or more isolates with either identical RFLP patterns (at least seven copies of IS6110) or identical RFLP and spoligotype patterns for isolates with RFLP patterns that had six or fewer copies of IS6110.

Differences in the proportion of TB patients from the TB genotyping network population living in cities with populations of $<100,000,100,001$ to $250,000,250,001$ to 500,000 , and $>500,000$ were compared with those of the national TB patients for the year 2000 only. Statistics were obtained from the U.S. Census Bureau (available at: URL: http://www.census.gov/ population/cen2000/phc-t6/tab04.pdf ).

Correlation of average TB incidence among cases at the seven sentinel sites and percentage of cases with isolates that clustered genetically were examined by year by using the Spearman rank correlation statistic. Clustering was determined by examining each year's cases independently. A MantelHaenszel chi-square or Fisher exact test was used, as appropriate, to ascertain whether the sentinel population was representative of TB patients in the United States in terms of demographic, clinical, behavioral, or outcome characteristics.

We used multiple logistic regression to assess the importance of demographic, clinical, behavioral, or outcome variables in predicting the occurrence of a given genotype for those genetic clusters that occurred most frequently ( $\geq 20$ isolates). The dependent variable was the presence or absence of a given genotype. The best-fit logistic regression model was determined by the strategy of Hosmer and Lemeshow (8). In brief, a univariate analysis of the categorical independent variables was done by using the Mantel-Haenszel chi-square or Fisher exact test, as appropriate; any variable with a significance value of $\geq 0.20$ was included in a best subset, multivariate logistic regression model. Collinearity of independent variables was assessed by using the variance/covariance matrix from PROC LOGISTIC (SAS Institute, Inc., Cary, NC) to generate condition indices and a matrix of variance decomposition proportions to detect dependencies among the vari- ables (9). Backward elimination of independent variables was performed if the probability of the independent variable was $\geq 0.20$. Both the Wald statistic and $95 \%$ confidence interval were used on each coefficient to assess the significance of variables in each model; the log-likelihood ratio was used to assess the overall significance of the final models, and the Hosmer-Lemeshow statistic was used to evaluate the fit of each of the final models. Data were analyzed by SAS version 8.0 software (SAS Institute, Inc.) (10).

\section{Results}

\section{Sentinel Population Characteristics}

The incidence of TB cases in the sentinel surveillance sites varied within and among sites over time (Table 1). From 1996 to 2000, the overall incidence of TB in the United States declined from 8.0 to 5.8 per 100,000 inhabitants, and similar downward trends were observed in each of the TB genotyping network sites. The California, New Jersey, Arkansas, and Texas sites had a higher incidence of TB than the overall national rates. The incidence rates in California and Texas (sites that included only six and four counties from each state) were similar to the overall incidence rates for each state (data not shown).

In the surveillance area, 15,035 patients with verified TB represented $16 \%$ of the TB patients in the United States during the 5-year study period (Table 2). Overall, 11,923 TB patients were culture-positive (721 from Arkansas, 2,842 from California, 1,192 from Maryland, 1,022 from Massachusetts, 1,481 from Michigan, 2,599 from New Jersey, and 2,066 from Texas). Of TB patients in the surveillance areas, $79.3 \%$ $(11,923)$ were culture positive, and RFLP results were available for $91.2 \%(10,883)$. However, spoligotyping results were not available for 131 of the isolates that had six or fewer copies of IS6110 $(5 \% ; n=2,638)$; thus, these patients were excluded from our analysis. Of 1,171 isolates not genotyped by RFLP or spoligotyping, 12 (1\%) were from Michigan, 35 (3\%) from Maryland, 40 (3\%) from Massachusetts, 110 (9\%)

\begin{tabular}{lcccccc}
\hline $\begin{array}{l}\text { Table 1. Incidence of tuberculosis cases in the United States and in } \\
\text { the sentinel surveillance areas of the National Tuberculosis Genotyp- } \\
\text { ing Surveillance Network, } \\
\text { 1996-2000 }\end{array}$ \\
\hline Sentinel surveillance site & 1996 & 1997 & 1998 & 1999 & 2000 & Mean \\
\hline Arkansas & 9.0 & 7.9 & 6.7 & 7.1 & 7.4 & 7.6 \\
California $^{b}$ & 16.3 & 13.9 & 13.9 & 12.9 & 11.6 & 13.7 \\
Maryland & 6.3 & 6.7 & 6.3 & 5.7 & 5.3 & 6.1 \\
Massachusetts & 4.3 & 4.4 & 4.6 & 4.4 & 4.5 & 4.4 \\
Michigan & 4.6 & 3.8 & 3.9 & 3.6 & 2.9 & 3.8 \\
New Jersey & 10.3 & 8.9 & 7.9 & 7.0 & 6.7 & 8.2 \\
Texas & 12.7 & 12.8 & 12.5 & 10.9 & 9.6 & 11.7 \\
United States & 8.0 & 7.4 & 6.8 & 6.4 & 5.8 & 6.9
\end{tabular}

${ }^{\mathrm{a}}$ Number per 100,000 inhabitants.

${ }^{\mathrm{b}}$ Sentinel surveillance areas for California and Texas did not include the entire states. 
from Arkansas, 156 (13\%) from Texas, 327 (28\%) from California, and 491 (42\%) from New Jersey. Primary reasons for lack of genotyping results included inability to obtain cultures from private health-care providers, contamination of cultures, or poorly growing or nonviable cultures.

Characteristics of the TB patient population from the genotyping network sentinel sites were comparable with those from the entire United States, with some exceptions (Table 2). Sentinel surveillance populations had higher proportions of women ( $42 \%$ for the genotyping network vs. $37 \%$ for the United States overall) and patients in the 15- to 44-year age category, and were more often homeless or lived in correctional or long-term care facilities. Higher proportions of genotyping network patients used intravenous drugs, but fewer patients used noninjecting drugs or alcohol excessively.

Of the study population, about $4 \%$ reported previous episodes of TB ( 652 of 15,035 ; Table 2$)$. Of persons with a previous recent history of TB, 28 had TB after completing $>1$ year of therapy within the study period; genotyping data on isolates from both episodes were available for 22 of these persons. A higher number of persons from the TB genotyping network study population lived within city limits $(97 \%$ vs. $87 \%)$. However, when compared with national averages, genotyping network populations were generally from smaller towns and cities: 1,446 (69\%) of 2,099 genotyping network patients were from cities and towns with $<250,000$ inhabitants, compared with 10,093 (62\%) of 16,377 TB patients nationwide (MantelHaenszel chi square $=41.8 ; \mathrm{p}<0.0001)$.

The proportion of foreign-born patients was higher in genotyping network populations compared with the overall national average ( $50 \%$ for genotyping network vs. $41 \%$ for the United States). Numbers of foreign-born TB patients increased over time at about the same rate for both genotyping network populations and national TB patients. From 1996 to 2000, national proportions of foreign-born TB patients increased from $37 \%(7,725 / 21,045)$ to $47 \%(7,593 / 16,281)$; in the genotyping network populations, the proportions of foreign-born TB patients increased from $44 \%(1,153 / 2,642)$ to $58 \%(1,222 /$ $2,092)$. Characteristics of the genotyping network population between sites were similar, as were culture-positive genotyping network populations compared with the overall genotyping network case population.

\section{Analysis of Genotyping Data}

The distribution and diversity of RFLP and spoligotyping pattern results from the genotyping network have been discussed in detail (11). In contrast to that analysis, we used both RFLP and spoligotyping results to define genetic clusters. Overall, 6,609 distinct patterns were identified, including 1,029 that contained $\geq 2$ isolates per cluster. When analyzed by site, 1,018 clusters were identified: 71 clusters were from Arkansas (611 cases genotyped, 2-16 cases per cluster), 233 from California (2,511 cases, 2-128 cases per cluster), 104 from Maryland (1,157 cases, 2-36 cases per cluster), 85 from Massachusetts (982 cases, 2-16 cases per cluster), 125 from
Michigan (1,469 cases, 2-102 cases per cluster), 196 from New Jersey (2,112 cases, 2-40 cases per cluster), and 204 from Texas (1,910 cases, $2-96$ cases per cluster). Overall, 970 distinct genotypes, including 235 representing clusters, had $\leq 6$ copies (2,507 cases, $24 \%$ clustered, $2-93$ cases per cluster). In contrast, 794 clusters from 5,639 distinct genotypes had $\geq 7$ IS6110 copies $(8,245$ cases, $14 \%$ clustered, $2-105$ cases per cluster). Most clusters included seven or fewer persons (85\%; 900/1,029).

\section{Longitudinal Analysis}

Most clusters occurred in only a single site $(66 \% ; 680 /$ $1,029)$. However, $260(25 \%)$ were found in two sites, $55(5 \%)$ in three sites, $19(2 \%)$ in four, $8(1 \%)$ in five, and $7(1 \%)$ in six sites. As expected, clusters that spanned multiple sites were larger. Clusters found at a single site averaged four persons per cluster (mean $=3.65$; standard error $[\mathrm{SE}] \pm 0.22 ; \mathrm{n}=680$ ), in contrast to 61 persons per cluster for the genotypes found at six sites (mean=61.14; $\mathrm{SE} \pm 23.6 ; \mathrm{n}=7$; Kruskal-Wallis test, $\mathrm{p}<0.0001)$. Most $(62 \%)$ of the 34 clusters that occurred in at least four sites occurred in all 5 years of the study; $26 \%$ in 4 years; and $6 \%$ each in 3 and 2 years of the study.

Changes in proportions of patients with isolates that clustered were observed over time. In the first 2 years of the study, the percentage of the cumulative total number of cases that clustered increased from $28 \%$ to $45 \%$; smaller increases occurred thereafter (Figure 1). Overall, the proportion of clustered cases was $48 \%(5,171 / 10,752)$. The percentages of clustered cases by sites were $28 \%(276 / 982)$ for Massachusetts; $34 \%(393 / 1,157)$ for Maryland; $41 \%(873 / 2,112)$ for New Jersey; $42 \%(1,046 / 2,511)$ for California; $44 \%(266 / 611)$ for Arkansas; 49\% $(720 / 1,469)$ for Michigan; and 57\% $(1,093 /$ $1,910)$ for Texas. Maximum cluster size and absolute numbers of cases with isolates that clustered continued to increase through the end of the study.

Overall, cases with isolates that clustered showed a concomitant decline with average incidence of TB over the 5-year period (Figure 2). A significant positive association was observed between the percentage of cases with clustered genotypes and TB incidence over time (Spearman $\mathrm{rho}=0.90$; $\mathrm{p}=0.037$ ).

\section{Risk Factor Analyses of Genetic Clusters}

Compared with persons whose isolates had unique genotypes, persons with isolates that clustered were more likely to be non-Hispanic, black men born in the United States. They were more likely to have pulmonary disease and abnormal chest radiographs with cavities; in addition, they more often had positive sputum smears; were HIV-positive, homeless, or residents of a correctional facility; and used drugs or alcohol excessively (Table 3 ). Patients with unclustered isolates were 5 years older on the average than those with isolates that clustered (44.8 years vs. 49.4 years, respectively; Table 3 ). Multiple logistic regression efforts resulted in models that were not robust (data not shown). 
TUBERCULOSIS GENOTYPING NETWORK

Table 2. Demographic and risk behavior factors and clinical, laboratory, and treatment outcomes for the sentinel surveillance patients (National Tuberculosis Genotyping and Surveillance Network) compared with factors and outcomes of all tuberculosis patients, United States, 1996-2000 ${ }^{\mathrm{a}, \mathrm{b}}$

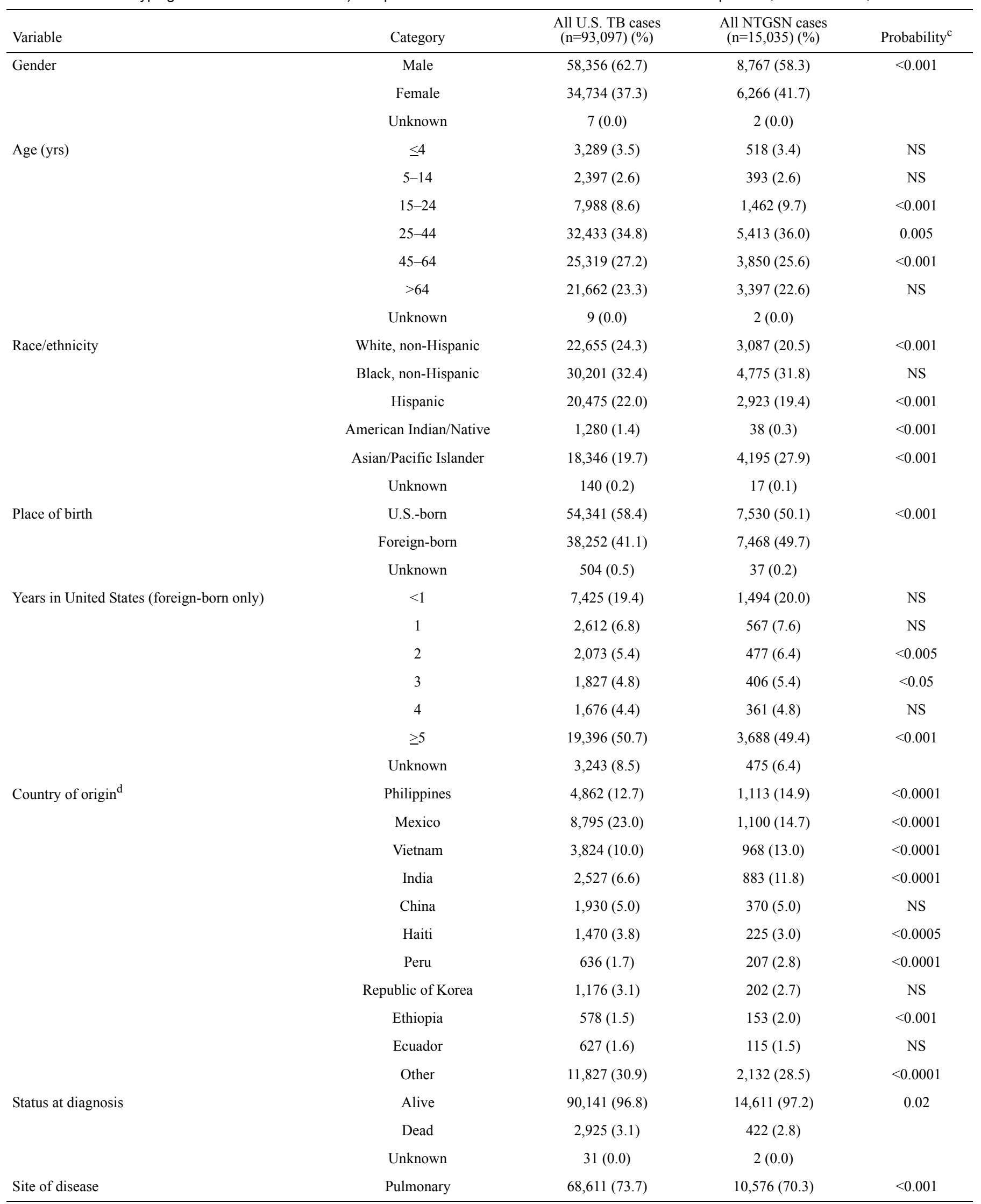


Table 2. (continued) Demographic and risk behavior factors and clinical, laboratory, and treatment outcomes for the sentinel surveillance patients (National Tuberculosis Genotyping and Surveillance Network) compared with factors and outcomes of all tuberculosis patients, United States, $1996-2000^{\mathrm{a}, \mathrm{b}}$

\begin{tabular}{|c|c|c|c|c|}
\hline Variable & Category & $\begin{array}{l}\text { All U.S. TB cases } \\
(\mathrm{n}=93,097)(\%)\end{array}$ & $\begin{array}{l}\text { All NTGSN cases } \\
(\mathrm{n}=15,035)(\%)\end{array}$ & Probability $^{\mathrm{c}}$ \\
\hline \multirow[t]{3}{*}{ Site of disease } & Extrapulmonary & $17,406(18.7)$ & $3,210(21.4)$ & $<0.001$ \\
\hline & Pulmonary and Extrapulmonary & $7,046(7.6)$ & $1,241(8.3)$ & 0.003 \\
\hline & Unknown & $34(0.0)$ & $8(0.1)$ & \\
\hline Primary disease site & Pulmonary & $73,157(78.6)$ & $11,365(75.6)$ & $<0.0001$ \\
\hline \multirow[t]{3}{*}{ Primary disease site } & Miliary & $1,407(1.5)$ & $241(1.6)$ & NS \\
\hline & All other & $10,345(11.1)$ & $1,727(11.5)$ & NS \\
\hline & Unknown & $34(0.0)$ & $8(0.0)$ & \\
\hline Sputum smear for acid-fast organisms & Negative & $36,912(39.6)$ & $5,995(39.9)$ & $<0.0001$ \\
\hline \multirow{2}{*}{ TST at diagnosis } & Positive & $54,113(58.1)$ & $8,799(58.5)$ & \\
\hline & Not done/unknown & $25,769(27.6)$ & $4,289(28.6)$ & \\
\hline \multirow[t]{4}{*}{ Case verification criteria } & Positive culture & $74,940(80.5)$ & $11,967(79.6)$ & $<0.01$ \\
\hline & Positive smear & $765(0.8)$ & $136(0.9)$ & NS \\
\hline & Clinical case & $11,286(12.1)$ & $1,858(12.4)$ & NS \\
\hline & Provider diagnosis & $6,106(6.6)$ & $1,074(7.1)$ & $<0.01$ \\
\hline \multirow[t]{2}{*}{ Chest radiograph ${ }^{\mathrm{e}}$} & Cavitary & $18,742(24.8)$ & $2,990(25.3)$ & NS \\
\hline & Noncavitary & $50,652(66.9)$ & $7,897(66.8)$ & NS \\
\hline \multirow{6}{*}{ HIV status ${ }^{\mathrm{f}}$} & Indeterminate & $47(0.1)$ & $6(0.1)$ & \\
\hline & Refused & $1,959(6.1)$ & $325(6.1)$ & \\
\hline & Not offered & $4,130(12.8)$ & $899(17.0)$ & \\
\hline & Test done, unknown & $714(2.2)$ & $115(2.2)$ & \\
\hline & Unknown & $2,812(8.7)$ & $658(12.4)$ & \\
\hline & Total & 32,249 & 5,293 & \\
\hline \multirow[t]{3}{*}{ Homeless within past year } & Yes & $5,789(6.2)$ & $646(4.3)$ & $<0.001$ \\
\hline & No & $84,873(91.2)$ & $14,185(94.3)$ & \\
\hline & Unknown & $2,435(2.6)$ & $204(1.4)$ & \\
\hline \multirow[t]{3}{*}{ Resident of correctional facility at diagnosis } & Yes & $3,352(3.6)$ & $377(2.5)$ & $<0.001$ \\
\hline & No & $89,479(96.1)$ & $14,617(97.2)$ & \\
\hline & Unknown & $266(0.3)$ & $41(0.3)$ & \\
\hline \multirow[t]{2}{*}{ Correctional facility type } & Federal prison & $164(4.9)$ & $6(1.6)$ & $<0.005$ \\
\hline & State prison & $1,036(30.9)$ & $97(25.7)$ & $<0.05$ \\
\hline
\end{tabular}


TUBERCULOSIS GENOTYPING NETWORK

Table 2. (continued) Demographic and risk behavior factors and clinical, laboratory, and treatment outcomes for the sentinel surveillance patients (National Tuberculosis Genotyping and Surveillance Network) compared with factors and outcomes of all tuberculosis patients, United States, $1996-2000^{\mathrm{a}, \mathrm{b}}$

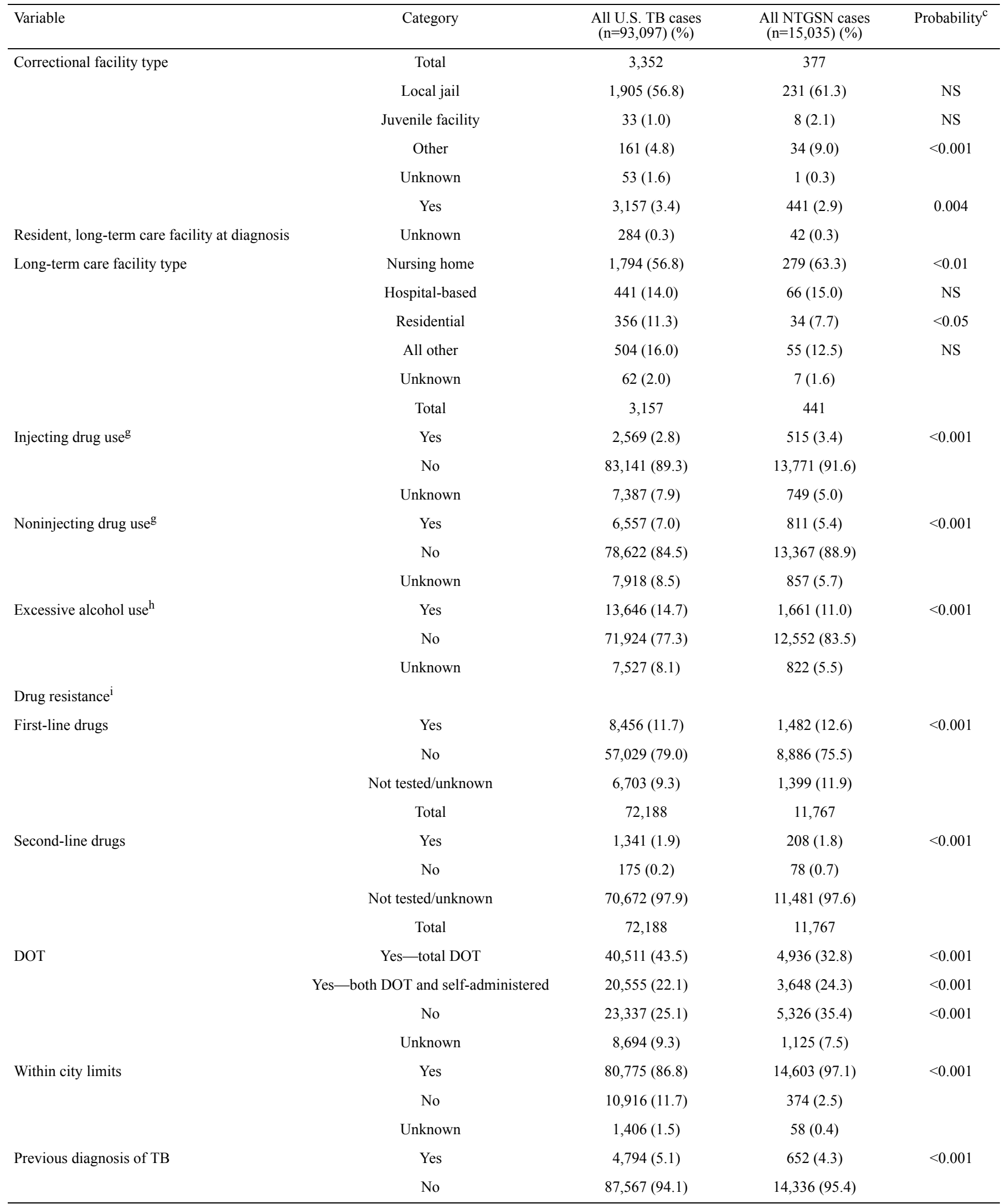


TUBERCULOSIS GENOTYPING NETWORK

Table 2. (continued) Demographic and risk behavior factors and clinical, laboratory, and treatment outcomes for the sentinel surveillance patients (National Tuberculosis Genotyping and Surveillance Network) compared with factors and outcomes of all tuberculosis patients, United States, $1996-2000^{\mathrm{a}, \mathrm{b}}$

\begin{tabular}{lccc}
\hline Variable & Category & All U.S. TB cases (n=93,097) (\%) & All NTGSN cases $(\mathrm{n}=15,035)(\%)^{\text {Probability }^{\mathrm{c}}}$ \\
\hline Previous diagnosis of TB & Unknown & $736(0.8)$ & $47(0.3$ \\
Duration of therapy (days) & Mean & 246 & 245 \\
& Median & 217 & 214 \\
& Std. dev. & 135 & 130 \\
& No. & 65,344 & 10,822 \\
\hline
\end{tabular}

${ }^{a}$ NTGSN, National Tuberculosis Genotyping Surveillance Network;TB, tuberculosis; DOT, directly observed therapy; TST, tuberculin skin test; Std. dev., standard deviation; NS, not significant $(\mathrm{p}>0.05)$

${ }^{\mathrm{b}}$ Subtotals for each category are listed if different from the total case numbers.

${ }^{\mathrm{c}}$ Probability of significant differences between U.S. TB patients and all NTGSN surveillance patients (chi-square test; t-test for duration of therapy); referent group is all other groups combined, excluding not done or unknown categories, unless otherwise noted.

${ }^{\mathrm{d}}$ Top 10 countries for foreign-born patients only.

excludes cases with extrapulmonary TB only.

${ }^{\mathrm{f}} \mathrm{HIV}$ cases from California are excluded because this site does not report HIV results on Report of a Verified Case of Tuberculosis forms; ages $15-44$ years only.

${ }^{\mathrm{g}}$ Injecting or noninjecting drug use within last year; includes use of licensed, prescription, or illegal drugs (not prescribed by a physician).

${ }^{h}$ Excessive use of alcohol within the past year as indicated by participation in alcohol treatment programs, diagnosis of alcoholism, or observation of intoxication during visits to health-care facilities.

${ }^{1}$ Drug resistance on initial testing of isolate. First-line drug resistance is resistance to at least one of the following: isoniazid, rifampin, ethambutol, or streptomycin. Second-line drug resistance is resistance to one or more of the following: ethionamide, kanamycin, cycloserine, capreomycin, para-amino salicylic acid, amikacin, rifabutin, ciprofloxacin, ofloxacin, or other drugs. Testing results for one or more of the drugs could have been missing.

Except for 4 genotypes, all 34 clusters with $\geq 20$ isolates per cluster had significant demographic, clinical, and behavioral risk factors (Table 4). Race, ethnicity, and place of birth were frequently significant predictors for a given genotype. Other predictors included gender, age, site of disease, resistance to first-line drugs, and alcohol or drug abuse (Table 4). Twelve (40\%) of 30 of these larger clusters were observed in four or more sites over a 5-year period. Lower percentages of foreign-born patients than U.S.-born patients clustered, regardless of the number of IS 6110 copies (Figure 3). More than 50\% $(1,025 / 1,825)$ of the foreign-born patients whose isolates clustered had been in the United States for $\geq 5$ years. Clustering of isolates from foreign-born patients ranged from 15\% (49/316) in Michigan to 38\% (309/816) in Texas.

\section{Discussion}

This population-based study is the largest that has been conducted in the United States to assess risk factors related to specific M. tuberculosis genotypes. Generally, clustered iso- lates have been considered recently acquired infections (12). However, this assumption may not always be correct. Clustering does not prove that transmission occurred, and its demonstration depends on adequate sampling of the population, incidence of TB, and characteristics of the study population (e.g., age structure, population mobility, duration of residence, and immune status) $(1,13)$. Only $25 \%-42 \%$ of patients in genetic clusters were shown to have epidemiologic connections with another member of the cluster (14-16). Conventional epidemiologic investigation of these TB patients (including interviews) was conducted, but inclusion in this analysis was outside the scope of this article. Thus, results that indicate clustered genotypes are representative of recent transmission should be interpreted with caution.

Given this caveat, our results nevertheless demonstrate several consistent patterns. Differences in demographic and other risk factors for persons with isolates that clustered corroborated those from smaller studies conducted in the United States and larger surveys in Europe. Extensive surveys from

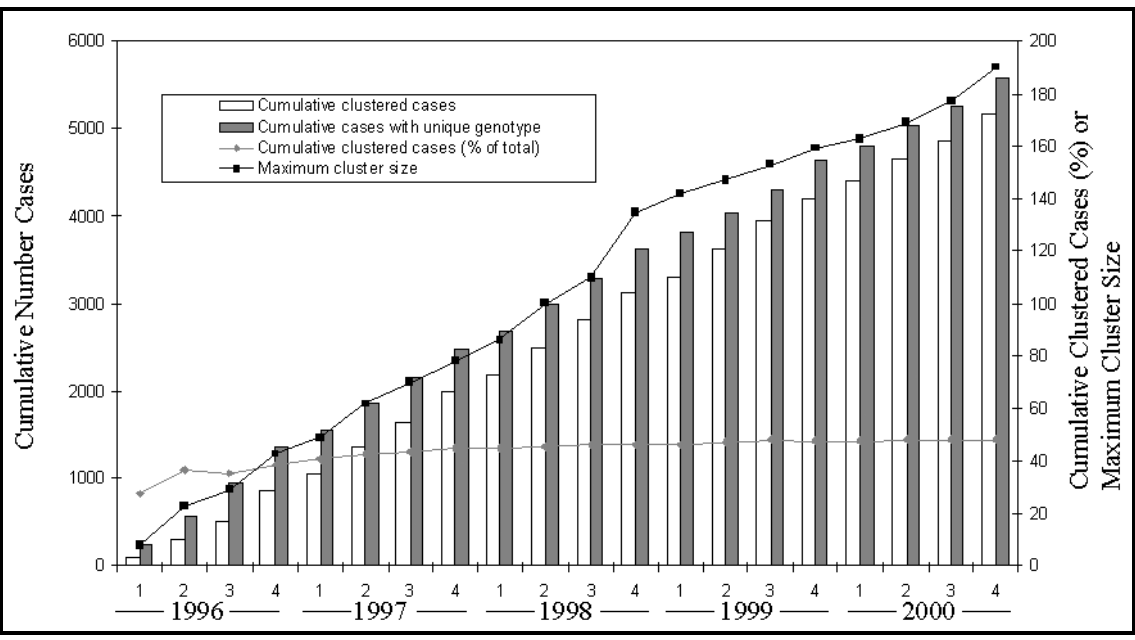

Figure 1. Numbers of tuberculosis cases, cumulative proportion of cases with isolates in genetic clusters, and maximum genetic cluster size from seven sentinel surveillance sites by quarter that verified case was counted, 1996-2000. Numbers of cases with isolates that had unique genotypes and those with isolates that were in genetic clusters are shown separately. 


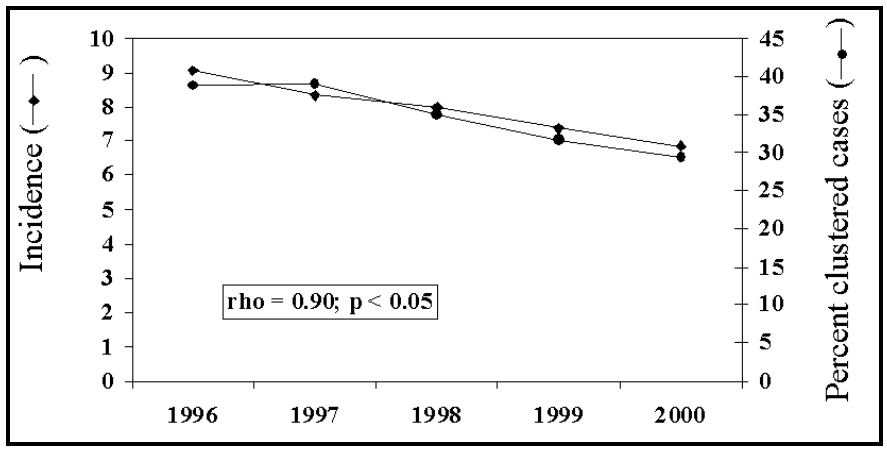

Figure 2. Average annual incidence of tuberculosis for seven sentinel surveillance sites and percentage of cases with isolates in genetic clusters, 1996 to 2000 . Spearman correlation coefficient and probability of correlation between incidence and percentage of cases clustered are given.

the Netherlands (17) also demonstrated that persons with isolates that clustered genetically were younger than those with unique genotypes. Other risk factors for clustering included being male, born in the United States, non-Hispanic black, or homeless; using drugs and alcohol excessively; and having pulmonary disease and cavitations on chest radiograph, a sputum smear with acid-fast bacilli, and HIV infection. These risk factors have been observed for TB patients in different communities $(12,18,19)$. The heterogeneity and diversity of the study population may account for our failure to produce a multivariate logistic model to predict clustering.

A third of the foreign-born cases were recent immigrants to the United States, and overall, the percentage of clustered isolates from foreign-born persons was lower than the percent- age from nonimmigrants (Figure 3), indicating that at least a portion of these cases resulted from reactivation of latent disease or recent infection in the country of origin. In addition, for foreign-born persons, clustering of M. tuberculosis increased with the duration of residence in the United States. These results suggest that recently imported strains of $M$. tuberculosis from foreign-born persons may not commonly spread to U.S. residents or that transmission may be occurring after a lag time before the imported strains manifest as disease in contacts. Similar observations have been published in studies from San Francisco, New York, Switzerland, and Norway (20-24). These data may also reflect gaps in our knowledge of M. tuberculosis genotypes in circulation; a comparison of the U.S. TB genotyping network results with other databases worldwide may be warranted.

Logistic regression analysis of the most commonly occurring strains demonstrated that different risk factors were associated with specific genotypes. Several genotypes were associated with ethnic origin (e.g., Asian or Pacific Islander and Hispanic patients with six and three genotypes, respectively; Table 4). A recent study in Norway showed that several clusters consisted of patients of the same ethnic origin (23). An association has also been observed between the patient's ethnic origin and IS6110 copy number (25). These results, in conjunction with additional epidemiologic data, may be useful in tracking the geographic origin and spread of M. tuberculosis strains of public health importance (26).

A small proportion of clustered isolates were from persons from more than four sites spanning 5 years of study (Table 4).

\begin{tabular}{|c|c|c|c|c|c|}
\hline Variable $^{\mathrm{b}}$ & & Clustered (\%) & Unclustered (\%) & Relative risk $(95 \% \mathrm{CI})$ & Probability $^{\mathrm{c}}$ \\
\hline Total cases $(n=10,752)$ & & $5.171(48.1)$ & $5,581(51.9)$ & & \\
\hline \multirow[t]{3}{*}{ Gender } & Male & $3,289(63.6)$ & $3,107(55.7)$ & $1.19(1.14 \%$ to $1.24 \%)$ & $<0.001$ \\
\hline & Female & $1,881(36.4)$ & $2,473(44.3)$ & & \\
\hline & Unknown & $1(0.0)$ & $1(0.0)$ & & \\
\hline Mean age (yrs; \pm S.E. $)$ & & $44.8( \pm 0.26)$ & $49.4( \pm 0.28)$ & & $<0.0001$ \\
\hline \multirow[t]{6}{*}{ Race/ethnicity } & White, non-Hispanic & $1,018(19.7)$ & $1,201(21.5)$ & $0.94(0.90 \%$ to $0.99 \%)$ & 0.02 \\
\hline & Black, non-Hispanic & $2,254(43.6)$ & $1,237(22.2)$ & $1.61(1.55 \%$ to $1.67 \%)$ & $<0.001$ \\
\hline & Hispanic & $914(17.7)$ & $1,112(19.9)$ & $0.92(0.88 \%$ to $0.97 \%)$ & 0.003 \\
\hline & American Indian/Native & $17(0.3)$ & $10(0.2)$ & & \\
\hline & Asian/Pacific Islander & $961(18.6)$ & $2,014(36.1)$ & $0.60(0.56 \%$ to $0.63 \%)$ & $<0.001$ \\
\hline & Unknown & $7(0.1)$ & $7(0.1)$ & & \\
\hline \multirow[t]{3}{*}{ Place of birth } & U.S.-born & $3,331(64.4)$ & $2,023(36.2)$ & $1.83(1.75 \%$ to $1.90 \%)$ & $<0.001$ \\
\hline & Foreign-born & $1,825(35.3)$ & $3,552(63.6)$ & & \\
\hline & Unknown & $15(0.3)$ & $6(0.1)$ & & \\
\hline \multirow[t]{3}{*}{ Recent arrival in United States ${ }^{\mathrm{d}}$} & Yes & $535(29.3)$ & $1,225(34.5)$ & $0.59(0.55 \%$ to $0.63 \%)$ & $<0.001$ \\
\hline & No & $1,181(64.7)$ & $2,111(59.4)$ & & \\
\hline & Unknown & $109(6.0)$ & $216(6.1)$ & & \\
\hline \multirow[t]{2}{*}{ Site of disease } & Pulmonary & $3,902(75.5)$ & $3,835(68.7)$ & $1.20(1.14 \%$ to $1.26 \%)$ & $<0.001$ \\
\hline & Extrapulmonary & $788(15.2)$ & $1,254(22.5)$ & $0.77(0.72 \%$ to $0.81 \%)$ & $<0.001$ \\
\hline
\end{tabular}


TUBERCULOSIS GENOTYPING NETWORK

Table 3. (continued) Comparison of demographic and behavioral risk factors and clinical and treatment outcomes of tuberculosis (TB) casepatients who have genetically clustered genotypes with factors and outcomes of patients who had unique genotype patterns ${ }^{\mathrm{a}}$

\begin{tabular}{|c|c|c|c|c|c|}
\hline Variable $^{\mathrm{b}}$ & & Clustered (\%) & Unclustered (\%) & Relative risk $(95 \% \mathrm{CI})$ & Probability ${ }^{\mathrm{c}}$ \\
\hline \multirow[t]{2}{*}{ Site of disease } & Pulmonary and extrapulmonary & $476(9.2)$ & $492(8.8)$ & & NS \\
\hline & Unknown & $5(0.1)$ & 0 & & \\
\hline \multirow{2}{*}{ Sputum smear } & Negative & $1,802(34.8)$ & $1,943(34.8)$ & & \\
\hline & Not done/unknown & $1,099(21.3)$ & $1,627(29.1)$ & & \\
\hline \multirow{4}{*}{ Chest radiograph $^{\mathrm{e}}$} & Noncavitary & $2,639(60.2)$ & $2,826(65.3)$ & & \\
\hline & Normal & $146(3.3)$ & $118(2.73)$ & & \\
\hline & Not done/unknown & $253(5.8)$ & $211(4.9)$ & & \\
\hline & Total & 4,383 & 4,327 & & \\
\hline \multirow[t]{5}{*}{ HIV status ${ }^{\mathrm{f}}$} & Positive & $458(22.2)$ & $223(11.8)$ & $1.37(1.29 \%$ to $1.46 \%)$ & $<0.001$ \\
\hline & Refused & $106(5.1)$ & $138(7.3)$ & & \\
\hline & Not offered & $252(12.2)$ & $354(18.7)$ & & \\
\hline & Unknown & $270(13.0)$ & $323(17.1)$ & & \\
\hline & Total & 2,064 & 1,889 & & \\
\hline \multirow[t]{3}{*}{ Homeless within past year } & Yes & $370(7.2)$ & $139(2.5)$ & $1.55(1.46 \%$ to $1.64 \%)$ & $<0.001$ \\
\hline & No & $4,724(91.4)$ & $5,370(96.2)$ & & \\
\hline & Unknown & $77(1.5)$ & $72(1.3)$ & & \\
\hline \multirow[t]{2}{*}{ Resident of correctional facility at diagnosis } & Yes & $190(3.7)$ & $69(1.2)$ & $1.55(1.43 \%$ to $1.67 \%)$ & $<0.001$ \\
\hline & No & $4,966(96.0)$ & $5,503(98.6)$ & & \\
\hline Noninjecting drug use ${ }^{\mathrm{g}}$ & Unknown & $376(7.3)$ & $301(5.4)$ & & \\
\hline \multirow[t]{3}{*}{ Excessive alcohol use ${ }^{g}$} & Yes & $948(18.3)$ & $371(6.6)$ & $1.61(1.54 \%$ to $1.67 \%)$ & $<0.001$ \\
\hline & No & $3,897(75.4)$ & $4,893(87.7)$ & & \\
\hline & Unknown & $326(6.3)$ & $317(5.7)$ & & \\
\hline \multirow[t]{5}{*}{ First-line drugs ${ }^{\mathrm{h}}$} & Yes & $622(12.1)$ & $755(13.7)$ & $0.93(0.87 \%$ to $0.99 \%)$ & 0.016 \\
\hline & No & $2,718(53.0)$ & $3,337(60.5)$ & & \\
\hline & Not done & $1,748(34.1)$ & $1,356(24.6)$ & & \\
\hline & Unknown & $45(0.9$ & $66(1.2)$ & & \\
\hline & Total & 5,133 & 5,514 & & \\
\hline
\end{tabular}

${ }^{\mathrm{a}} \mathrm{CI}$, confidence interval; S.E., standard error.

${ }^{b}$ Only factors that had significant differences are shown.

${ }^{\mathrm{c}}$ Probability of chi-square statistic is shown, except for t-test results from analysis of age from each group.

${ }^{\mathrm{d}}$ Foreign-born only; arrived in the United States within 2 years.

${ }^{\text {e}}$ Excludes cases with extrapulmonary TB only.

${ }^{\mathrm{f}}$ California TB cases not included; ages $15-44$ years only.

${ }^{\mathrm{g}}$ Excessive drug or alcohol use within last year.

${ }^{h}$ First-line drug resistance is resistance to at least one of the following: isoniazid, rifampin, ethambutol, or streptomycin. Second-line drug resistance is resistance to one or more of the following: ethionamide, kanamycin, cycloserine, capreomycin, para-amino salicylic acid, amikacin, rifabutin, ciprofloxacin, ofloxacin, or other drugs. Testing results for one or more of the drugs could have been missing. 
TUBERCULOSIS GENOTYPING NETWORK

Table 4. Odds ratios from best-fit logistic regression analyses of the presence or absence of a specific genetic cluster of Mycobacterium tuberculosis on demographic, clinical, behavioral, or treatment outcome variables ${ }^{\text {a }}$

\begin{tabular}{|c|c|c|c|c|c|c|}
\hline Designation $^{\mathrm{c}}$ & IS6110 copies & Spoligotype $^{\mathrm{c}}$ & $\mathrm{N}$ & Main effect & Odds ratio estimates $(95 \% \mathrm{CI})^{\mathrm{b}}$ & Wald $\mathrm{p}^{\mathrm{b}}$ \\
\hline \multirow[t]{2}{*}{$00003^{c}$} & 1 & 777777777760771 & 40 & Asian/Pacific Islander & $3.70(1.51 \%$ to $9.02 \%)$ & 0.004 \\
\hline & & & & Age & $0.98(0.96 \%$ to $0.99 \%)$ & 0.017 \\
\hline \multirow[t]{2}{*}{$00129^{\mathrm{d}}$} & 1 & 777777777413771 & 25 & Asian/Pacific Islander & $73.3(17.0 \%$ to $315.6 \%)$ & $<0.0001$ \\
\hline & & & & Extrapulmonary infection & $2.57(1.10 \%$ to $6.03 \%)$ & 0.03 \\
\hline $00129^{d}$ & & & & Foreign-born & $10.4(1.55 \%$ to $70.12 \%)$ & 0.02 \\
\hline \multirow[t]{2}{*}{$00129^{d}$} & 1 & 777777777413731 & 13 & Asian/Pacific Islander & $13.88(3.71 \%$ to $51.92 \%)$ & $<0.0001$ \\
\hline & & & & Resistance to first-line drugs ${ }^{\mathrm{d}}$ & $3.80(1.22 \%$ to $11.86 \%)$ & 0.02 \\
\hline 00129 & 1 & 777776407760601 & 40 & Female & $2.73(1.43 \%$ to $5.23 \%)$ & 0.0025 \\
\hline 00016 & & & & Black, non-Hispanic & $10.88(5.48 \%$ to $21.6 \%)$ & 0.006 \\
\hline $00016^{\mathrm{c}}$ & 2 & 777776777760771 & 82 & Hispanic & $16.36(10.15 \%$ to $26.37 \%)$ & $<0.0001$ \\
\hline \multirow[t]{3}{*}{00016} & 2 & 037776777760601 & 30 & Age & $1.03(1.01 \%$ to $1.05 \%)$ & 0.006 \\
\hline & & & & Black, non-Hispanic & $7.13(2.36 \%$ to $21.53 \%)$ & 0.0005 \\
\hline & & & & Resident, long-term care facility & $3.67(1.17 \%$ to $11.70 \%)$ & 0.026 \\
\hline \multirow[t]{2}{*}{$00016^{\mathrm{d}}$} & 2 & 777776777760601 & 175 & U.S.-born & $3.12(1.85 \%$ to $5.26 \%)$ & $<0.0001$ \\
\hline & & & & Excessive alcohol use & $0.55(0.37 \%$ to $0.83 \%)$ & 0.0048 \\
\hline \multirow[t]{2}{*}{00370} & 3 & 700036777760731 & 13 & White, non-Hispanic & $5.20(1.52 \%$ to $17.79 \%)$ & 0.0087 \\
\hline & & & & HIV positive & $5.87(1.69 \%$ to $20.41 \%)$ & 0.005 \\
\hline 00015 & & & & Excessive alcohol use & $2.28(1.02 \%$ to $5.13 \%)$ & 0.05 \\
\hline \multirow[t]{2}{*}{00768} & 9 & & 19 & Black, non-Hispanic & $11.68(1.54 \%$ to $88.87 \%)$ & 0.02 \\
\hline & & & & Noninjecting drug use & $2.77(1.11 \%$ to $6.92 \%)$ & 0.03 \\
\hline \multirow[t]{5}{*}{$00242^{\mathrm{d}}$} & 10 & & 95 & Male & $2.12(1.27 \%$ to $3.56 \%)$ & 0.004 \\
\hline & & & & Age & $0.97(0.96 \%$ to $0.98 \%)$ & $<0.0001$ \\
\hline & & & & U.S.-born & $8.44(2.63 \%$ to $27.09 \%)$ & 0.0003 \\
\hline & & & & Homeless & $3.60(2.16 \%$ to $5.98 \%)$ & $<0.0001$ \\
\hline & & & & Noninjecting drug use & $0.46(0.24 \%$ to $0.90 \%)$ & 0.02 \\
\hline 00028 & 11 & & 70 & Black, non-Hispanic & $17.57(5.50 \%$ to $56.12 \%)$ & $<0.0001$ \\
\hline 00159 & 11 & & 24 & Excessive alcohol use & $2.76(1.23 \%$ to $6.22 \%)$ & 0.01 \\
\hline \multirow[t]{2}{*}{00325} & 11 & & 20 & Age & $1.03(1.01 \%$ to $1.06 \%)$ & 0.01 \\
\hline & & & & Excessive alcohol use & $3.08(1.22 \%$ to $7.70 \%)$ & 0.02 \\
\hline 00673 & 11 & & 25 & Asian/Pacific Islander & $84.6(19.85$ to $361.9 \%)$ & $<0.0001$ \\
\hline \multirow[t]{2}{*}{00757} & 11 & & 16 & Age & $0.90(0.85 \%$ to $0.94 \%)$ & $<0.0001$ \\
\hline & & & & HIV positive & $4.86(1.60 \%$ to $14.79 \%)$ & 0.005 \\
\hline
\end{tabular}


TUBERCULOSIS GENOTYPING NETWORK

Table 4. (continued) Odds ratios from best-fit logistic regression analyses of the presence or absence of a specific genetic cluster of Mycobacterium tuberculosis on demographic, clinical, behavioral, or treatment outcome variables ${ }^{\text {a }}$

\begin{tabular}{|c|c|c|c|c|c|c|}
\hline Designation $^{\mathrm{c}}$ & IS6110 copies & Spoligotype $^{\mathrm{c}}$ & $\mathrm{N}$ & Main effect & Odds ratio estimates $(95 \% \mathrm{CI})^{\mathrm{b}}$ & Wald $p^{b}$ \\
\hline \multirow[t]{2}{*}{$00019^{c}$} & 12 & & 27 & Male & $3.68(1.10 \%$ to $12.39 \%)$ & 0.03 \\
\hline & & & & White, non-Hispanic & $5.4(2.35 \%$ to $11.08 \%)$ & $<0.0001$ \\
\hline 00372 & & & & Resident, long-term care facility & $5.52(1.535$ to $20.0 \%)$ & 0.009 \\
\hline 00035 & 13 & & 33 & Black, non-Hispanic & $6.96(2.3 \%$ to $21.0 \%)$ & 0.0006 \\
\hline 00867 & & & & Noninjecting drug use & $2.77(1.11 \%$ to $6.92 \%)$ & 0.03 \\
\hline \multirow[t]{2}{*}{01284} & 17 & & 46 & Black, non-Hispanic & $2.40(1.22 \%$ to $3.57 \%)$ & $<0.0001$ \\
\hline & & & & Pulmonary disease & $0.92(-0.01 \%$ to $1.86 \%)$ & 0.054 \\
\hline $00237^{\mathrm{c}}$ & 21 & & 98 & White, non-Hispanic & $2.80(1.81 \%$ to $4.33 \%)$ & $<0.0001$ \\
\hline 01693 & & & & Extrapulmonary disease & $3.99(1.69,9.42)$ & 0.002 \\
\hline \multirow[t]{2}{*}{00027} & 22 & & 78 & Black, non-Hispanic & $1.74(1.05 \%$ to $2.90 \%)$ & 0.03 \\
\hline & & & & Sputum-smear positive & $3.07(1.75 \%$ to $5.39 \%)$ & $<0.0001$ \\
\hline
\end{tabular}

${ }^{\mathrm{a}} \mathrm{CI}$, confidence interval.

${ }^{b}$ Only genetic clusters that had $\geq 20$ isolates were included in the analysis; some samples sizes are $<20$ because of missing data among independent variables (Wald $95 \%$ confidence intervals given in parentheses). Only genetic clusters with significant predictors are listed. Age was modeled as a continuous variable.

${ }^{\mathrm{c}}$ The National Tuberculosis Genotyping Surveillance Network (NTGSN) designation for the IS6110 RFLP pattern is represented; spoligotype octal code designations are presented only for those genetic clusters from isolates that had $\leq 6$ copies of IS6110. RFLP patterns and spoligotypes are detailed elsewhere (11).

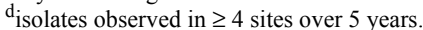

${ }^{\mathrm{e}}$ First-line drug resistance is resistance to at least one of the following: isoniazid, rifampin, ethambutol, or streptomycin. Second-line drug resistance is resistance to one or more of the following: ethionamide, kanamycin, cycloserine, capreomycin, para-amino salicylic acid, amikacin, rifabutin, ciprofloxacin, ofloxacin, or other drugs.

Although an in-depth analysis of epidemiologic links was not possible in this study, we found no evidence of recent transmission between patients with identical genotypes from the different states (data not shown); this lack of transmission was also noted in a smaller study in the United States (27). Since TB transmission is generally considered a local event, these ubiquitous genotypes may be widespread because of social factors (e.g., homelessness or alcohol or drug abuse; Table 4). In addition, these genotypes may represent older, endemic domestic strains that have been in the United States for centuries and have dispersed more widely throughout the United States than the more recently imported strains. Further molecular characterization of these genotypes may show additional differences not detected by RFLP. Nonetheless, the effect of M. tuberculosis virulence or host factors on the distribution of these genotypes cannot be ascertained.

The proportion of strains that were classified into clusters of identical genotypes (48\%) was comparable with proportions in the Netherlands and Denmark $(50 \%)(2,28)$, but the proportion was considerably higher than in two other countries $(17 \%$ in Switzerland [29]; 20\% in Norway [23]). The cumulative percentage of clustered strains reached a plateau by the end of the study's second year (Figure 1), a finding consistent with other molecular epidemiologic TB studies (2). Increases in maximum cluster size were anticipated because, as sample sizes increase with time, the number of isolates in each cluster would be expected to increase. In addition, higher proportions of clustered cases were observed for low-band number patterns (Figure 3), which had the maximum cluster size and may

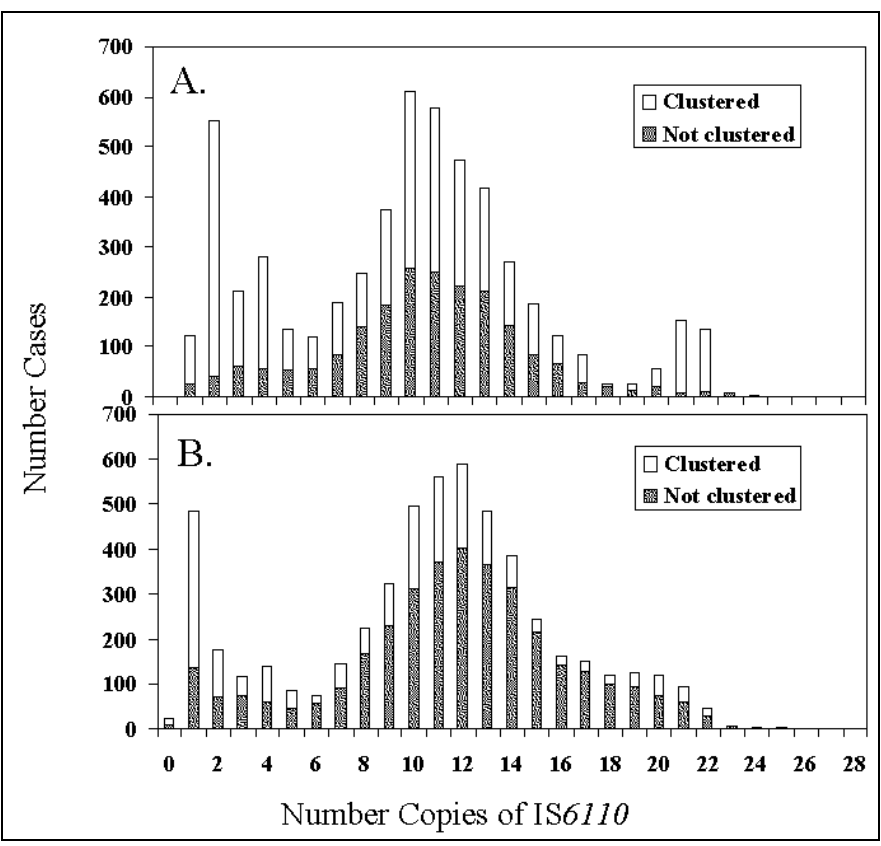

Figure 3. Number of cases with isolates that had unique genotypes ("not clustered") and those in genetic clusters for U.S.-born (A) and foreign-born persons (B) by number of copies of IS6110. 
indicate that the low-copy IS6110 patterns are not specific, even with the addition of spoligotyping.

The sensitivity and specificity of IS6110 RFLP in molecular epidemiologic studies have not been quantified and represent a potential limitation of this study. Although the stability of IS6110 is relatively high, the half-life of IS6110 RFLP is estimated to be 3-10 years (29-31) based on typing of serial isolates from individual patients. A study of isolates from patients in confirmed chains of transmission showed little change in IS6110 patterns (32). Calculation of these rates may be influenced by the duration between time of disease onset and time of sampling and may be proportional to the effectiveness of the TB control program (30). Because genotyping results were not available for $10 \%$ of TB cases in this study, estimates of the degree of clustering and the size of clusters are conservative. Some unique isolates might have clustered if some of the missing isolates had been available or if other cases with the same strain were present outside the study area (33).

Sentinel surveillance sites defined by artificial boundaries (i.e., state lines) not entirely representative of TB patients from the United States were included in this study. More than 90\% of the isolates from patients from the surveillance areas were genotyped, and these isolates were representative of those culture-positive patients from the sentinel surveillance areas. However, $16 \%$ of all TB case-patients reported in the United States were included in these sentinel surveillance sites during the 5-year study period. In addition, the sentinel surveillance population had higher proportions of foreign-born persons than the national average. Because of the propensity of foreign-born persons to have isolates with unique genotypes, the actual rate of clustering may have been underestimated. Nonetheless, sentinel surveillance of TB cases has provided a useful method for documenting genotypes in circulation in the United States and for identifying risk factor correlates of common genotypes.

Annual declines in TB incidence were paralleled by similar declines in the proportion of cases with genotypes in clusters (Figure 2), a finding consistent with the hypothesis that decreased clustering is expected with declining incidence (20). Since effort was similar each year, this association is not likely to be an artifact related to sample size (i.e., as sample size or number of cases becomes smaller, the probability of detecting clusters decreases). These findings underscore the importance of long-term longitudinal molecular studies and the potential usefulness of these methods in evaluating program effectiveness and improving program management.

\section{Acknowledgments}

We thank Ida Onorato, Ken Castro, Tom Shinnick, and Thomas Navin for their scientific guidance and logistic support; Elsa Villarino and James Mills for valuable comments on an earlier version of the manuscript; Annie Faye Prescott for excellent editorial assistance; and the health officials at local and state TB control offices that supported the activities of the National Tuberculosis Genotyping and Surveillance Network.

\section{References}

1. Foxman B, Riley L. Molecular epidemiology: focus on infection. Am J Epidemiol 2001;153:1135-41.

2. van Soolingen D. Molecular epidemiology of tuberculosis and other mycobacterial infections: main methodologies and achievements. J Intern Med 2001;249:1-26.

3. van Embden JD, van Soolingen D, Small PM, Hermans PW. Genetic markers for the epidemiology of tuberculosis. Res Microbiol 1992;143:385-91.

4. Fletcher HA. Molecular epidemiology of tuberculosis: recent developments and applications. Curr Opin Pulm Med 2001;7:154-9.

5. Castro KG, Jaffe HW. Rationale and methods for the National Tuberculosis Genotyping and Surveillance network. Emerg Infect Dis 2002; 8;1188-91.

6. Crawford JT, Braden CR, Schable BA, Onorato IM. National Tuberculosis Genotyping and Surveillance network: design and methods. Emerg Infect Dis 2002;8;1192-6.

7. Centers for Disease Control and Prevention. Tuberculosis information management system (TIMS) user's guide. Surveillance appendix I. Atlanta: U.S. Department of Health and Human Services, Public Health Service; 1998. p. 68.

8. Hosmer DW, Lemeshow S. Applied logistic regression. New York: John Wiley and Sons, Inc.; 1989.

9. Davis CE, Hyde JE, Bangdiwala SI, Nelson JJ. An example of dependencies among variables in a conditional logistic regression. In: Moolgavkar $\mathrm{S}$, Prentice RL, editors. Modern statistical methods in chronic disease epidemiology. New York: John Wiley and Sons, Inc.; 1986. p. 140-7.

10. SAS/STAT User's Guide. Cary, NC: SAS Institute Inc.; 2000.

11. Cowan LS, Crawford JT. Genotype analysis of Mycobacterium tuberculosis isolates from a sentinel surveillance population. Emerg Infect Dis 2002;8;1294-1302.

12. Small PM, Hopewell PC, Singh SP, Paz A, Parsonnet J, Ruston DC, et al. The epidemiology of tuberculosis in San Francisco: a population-based study using conventional and molecular methods. N Engl J Med 1994;330:1703-9.

13. Jasmer RM, Hahn JA, Small PM, Daley CL, Behr MA, Moss AR, et al. A molecular epidemiologic analysis of tuberculosis trends in San Francisco, 1991-1997. Ann Intern Med 1999;130:971-8.

14. Lockman S, Sheppard JD, Braden CR, Mwasekaga MJ, Woodley CL, Kenyon TA, et al. Molecular and conventional epidemiology of $\mathrm{Myco}$ bacterium tuberculosis in Botswana: a population-based prospective study of 301 pulmonary tuberculosis patients. J Clin Microbiol 2001;39:1042-7.

15. Braden CR, Templeton GL, Cave MD, Valway S, Onorato IM, Castro $\mathrm{KG}$, et al. Interpretation of restriction fragment length polymorphism analysis of Mycobacterium tuberculosis isolates from a state with a large rural population. J Infect Dis 1997;175:1446-52.

16. Frieden TR, Woodley CL, Crawford JT, Lew D, Dooley SM. The molecular epidemiology of tuberculosis in New York City: the importance of nosocomial transmission and laboratory error. Tuber Lung Dis 1996;77:407-13.

17. Vynnycky E, Nagelkerke N, Borgdorff MW, van Soolingen D, van Embden JD, Fine PE. The effect of age and study duration on the relationship between "clustering" of DNA fingerprint patterns and the proportion of tuberculosis disease attributable to recent transmission. Epidemiol Infect 2001;126:43-62.

18. Alland D, Kalkut GE, Moss AR, McAdam RA, Hahn JA, Bosworth W, et al. Transmission of tuberculosis in New York City. An analysis by DNA fingerprinting and conventional epidemiologic methods. N Engl J Med 1994;330:1710-6.

19. Friedman CR, Quinn GC, Kreiswirth BN, Perlman DC, Salomon N, Schluger N, et al. Widespread dissemination of a drug-susceptible strain of Mycobacterium tuberculosis. J Infect Dis 1997;176:478-84.

20. Borgdorff MW, Behr MA, Nagelkerke NJ, Hopewell PC, Small PM. Transmission of tuberculosis in San Francisco and its association with immigration and ethnicity. Int J Tuberc Lung Dis 2000;4:287-94. 
21. Geng E, Kreiswirth B, Driver C, Li J, Burzynski J, DellaLatta P, et al. Changes in the transmission of tuberculosis in New York City from 1990 to 1999. N Engl J Med 2002;346:1453-8.

22. Tornieporth NG, Ptachewich Y, Poltoratskaia N, Ravi BS, Katapadi M, Berger JJ, et al. Tuberculosis among foreign-born persons in New York City, 1992-1994: implications for tuberculosis control. Int J Tuberc Lung Dis 1997;1:528-35.

23. Dahle UR, Sandven P, Heldal E, Caugant DA. Molecular epidemiology of Mycobacterium tuberculosis in Norway. J Clin Microbiol 2001;39:1802-7.

24. Genewein A, Telenti A, Bernasconi C, Mordasini C, Weiss S, Maurer AM, et al. Molecular approach to identifying route of transmission of tuberculosis in the community. Lancet 1993;342:841-4.

25. Park YK, Bai GH, Kim SJ. Restriction fragment length polymorphism analysis of Mycobacterium tuberculosis isolated from countries in the western pacific region. J Clin Microbiol 2000;38:191-7.

26. Bifani PJ, Plikaytis BB, Kapur V, Stockbauer K, Pan X, Lutfey ML, et al. Origin and interstate spread of a New York City multidrug-resistant Mycobacterium tuberculosis clone family. JAMA 1996;275:452-7.

27. Yang Z, Barnes PF, Chaves F, Eisenach KD, Weis SE, Bates JH, et al. Diversity of DNA fingerprints of Mycobacterium tuberculosis isolates in the United States. J Clin Microbiol 1998;36:1003-7.

28. Bauer J, Yang Z, Poulsen S, Andersen AB. Results from 5 years of nationwide DNA fingerprinting of Mycobacterium tuberculosis complex isolates in a country with a low incidence of $M$. tuberculosis infection. J Clin Microbiol 1998;36:305-8.
29. Pfyffer GE, Strassle A, Rose N, Wirth R, Brandli O, Shang H. Transmission of tuberculosis in the metropolitan area of Zurich: a 3 year survey based on DNA fingerprinting. Eur Respir J 1998;11:804-8.

30. de Boer AS, Borgdorff MW, de Haas PE, Nagelkerke NJ, van Embden JD, van Soolingen D. Analysis of rate of change of IS6110 RFLP patterns of Mycobacterium tuberculosis based on serial patient isolates. J Infect Dis 1999;180:1238-44.

31. Warren RM, van der Spuy GD, Richardson M, Beyers N, Borgdorff MW, Behr MA, et al. Calculation of the stability of the IS 6110 banding pattern in patients with persistent Mycobacterium tuberculosis disease. J Clin Microbiol 2002;40:1705-8.

32. Niemann S, Rusch-Gerdes S, Richter E, Thielen H, Heykes-Uden H, Diel R. Stability of IS6110 restriction fragment length polymorphism patterns of Mycobacterium tuberculosis strains in actual chains of transmission. J Clin Microbiol 2000;38:2563-7.

33. Murray M, Alland D. Methodological problems in the molecular epidemiology of tuberculosis. Am J Epidemiol 2002;155:565-71.

Address for correspondence: Barbara Ellis, National Center for Infectious Diseases, Centers for Disease Control and Prevention, Mailstop E79, Atlanta, GA 30333, USA; fax: 404-498-2270; e-mail: bae7@cdc.gov

\section{EMERGING \\ Full text free online at www.cdc.gov/eid}

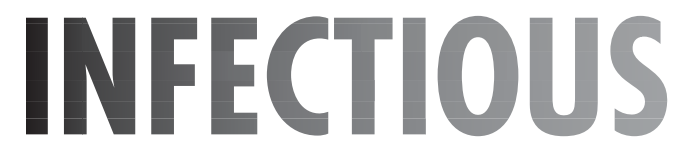

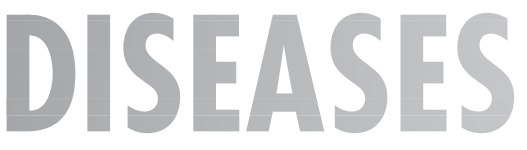

The print journal is available at no charge to public health professionals

YES, I would like to receive Emerging Infectious Diseases.

Please print your name and business address in the box and return by fax to $404-371-5449$ or mail to

\section{EID Editor}

CDC/NCID/MS D61

1600 Clifton Road, NE

Atlanta, GA 30333

Moving? Please give us your new address (in the box) and print the number of your old mailing label here

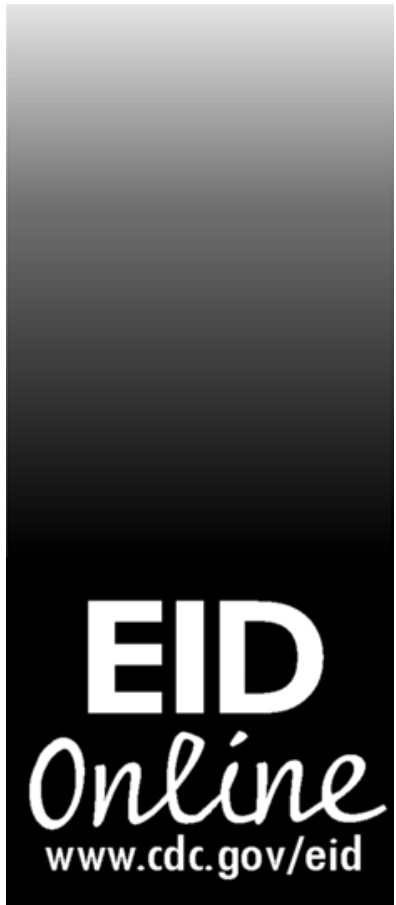

